\title{
Staff training in integrated sexual health services
}

\section{R Kane, K Wellings}

\section{Coordination of family planning and GUM services has the potential to boost the effectiveness of both}

T he move towards integration of family planning and genitourinary medicine (GUM) services (that is, the reorganisation of the two specialties, strategically and in terms of setting, in a more client centred approach) has recently been gathering momentum in the United Kingdom. Providers of family planning services are increasingly being urged to adopt a broader remit in respect of their clients' needs for STI and HIV prevention and treatment, ${ }^{1}$ as are providers of STI services in respect of their clients' contraceptive needs. Although fully integrated sexual health services are still comparatively rare, a growing number of family planning services provide STI diagnosis and treatment and a growing number of GUM clinics provide contraceptive services. ${ }^{2}{ }^{3}$ The trend may be accelerated as a result of the government's sexual health strategy, ${ }^{4}$ in England. The systematic evaluation of three pilot "one stop shops" is likely to provide more robust evidence on the acceptability and effectiveness of integrated services which, if favourable, could provide the impetus to further expansion.

In theory, the case for an integrated approach to sexual health service provision is compelling. Control of fertility and of sexually transmitted infection share common characteristics. The choice of contraceptive method may have implications for transmission of infectious agents and the presence of infection has consequences for fertility. The two services also share potential clients. Studies of GUM clinic attenders have shown a high proportion to be at risk of pregnancy ${ }^{5-9}$ and studies of family planning clinics attenders show sizeable proportions to be at risk of infection..$^{1011}$ There is also evidence that service users prefer a one stop service providing coordinated sexual health care. ${ }^{71213}$ Advocates of integration claim that coordination of family planning and GUM services has the potential to boost the effectiveness of both, broadening opportunities for screening and prevention, reducing duplication of services, providing continuity of care, and making maximum use of finite resources. ${ }^{2}{ }^{14-16}$

The shift towards a broader remit has, however, clear implications for training of personnel working in each of the two specialties. ${ }^{1}$ Broader based training is seen as an essential prerequisite to more holistic provision. Healthcare professionals in each of the specialties need at least a basic knowledge and skills of the other in order to provide a holistic approach to sexual health care. ${ }^{17}$ Insecurities relating to the prospect of taking on new working roles are commonly attributed to limitations of previous training and experience.

Until recently, training has not been conducive to integration. ${ }^{15}{ }^{18}$ Anxieties have been expressed that the move towards integrated care may be hindered by resistance from clinicians constrained by their training background. ${ }^{19}$ The evolution of integrated services clearly needs to take place in parallel with the training of those providing them. There are those who fear that the training plans may be jumping the gun, and that the organisational structures should perhaps remain unchanged until such a time that the staff are well enough equipped to run new integrated units. Yet the more commonly held view is that training is lagging behind, and so hindering the development of an integrated service. ${ }^{17}$ The move towards integration has been progressing faster than has progress towards the staff training needed to underpin it and this has been seen by some as a barrier to introducing STI services into family planning. ${ }^{1}$

\section{Studies of GUM clinic attenders have shown a high proportion to be at risk of pregnancy and studies of family planning clinics attenders show sizeable proportions to be at risk of infection}

Despite recognition of the importance of a wider knowledge of sexual health to successful working in integrated services, in-depth coverage of each specialty is not yet part of current undergraduate medical training. Similarly, family planning trained nurses working in existing integrated services, together with more recent recruits, are likely to have received a broader training, yet many who have practised for some time have no GU training. Community family planning services are increasingly taking on a role in the diagnosis and treatment of STIs, but deficiencies with regard to the diagnosis and management of STIs have led to recommendations for better training for family planning staff. ${ }^{10}$

As to how training needs might be met, there is not a great deal of consensus. Committed advocates of integrated services are more likely to believe in the need for comprehensive dual training so that staff would be fully trained in both fields. According to this perspective, a new breed of consultants, among whom the divide between the specialties is apparent in neither training nor background, is the model for the future. There are concerns however, on how well equipped practitioners will feel to manage the more complicated aspects of patient care in both disciplines. Others question whether full dual training is a realistic or even a necessary goal, particularly in the short term. ${ }^{17}$ Training needs are likely to be burdensome, since each of the two areas have traditionally been organised as total specialties in themselves, and coverage of both in their entirety is likely to be taxing. The training workload of GUM has in itself increased since the advent of HIV and additional training in contraception may simply be too onerous. Similarly, expecting all FP staff to undertake extensive training in HIV seems unreasonable.

A modular approach may be the answer, combining basic core training to enable those working in one specialty to provide care in the other, with higher level training to equip specialised practitioners to deal with less run of the mill problems. In practice, this would result in a two tier system; two physicians would work in parallel, with considerable overlap between them in terms of basic skills, but with some exclusive areas of expertise in which each had specialised training. Having the two services under one roof is likely to facilitate such an approach, but collaboration and cooperation between sites is likely to achieve similar ends.

For existing professionals, the attention is on update training and here the challenges relate to the practical issues of finding time and resources. Staffing levels may not permit time off work for attendance at training courses. GU trained nurses would gain from the opportunity to acquire practical experience in 


\section{Key messages}

- The move towards the integration of family planning and GUM services, has been progressing faster than has progress towards the staff training needed to underpin it.

- There is little consensus as to how training needs might best be met; the optimal way of ensuring FP and GUM staff are equipped to work across specialties needs further debate.

- Staff are subjected to many practical barriers to training, principally time and cost.

- The difference in the structure and culture of family planning and GUM and the perceived status inequalities which seem to stem from these differences, constitute other, more fundamental barriers to training.

- Training is likely to be an important determinant, not merely of the comprehensiveness of service provision, but also of the cohesiveness of the profession.

family planning clinics, yet relatively fewer opportunities for observation exist in the context of family planning, compared with GUM. Student attachments are rarer in family planning settings, problems of confidentiality may be more pronounced, and some health professionals are concerned that family planning staff are not equipped to train. ${ }^{17}$

Barriers may also exist at service level. For example, services often have to undergo significant restructuring to cope with increasing client demand. One current example is the move towards the more advanced roles of nursing staff. Concurrent developments such as these also require fundamental restructuring and the implementation of new procedures and protocols, and may themselves create additional obstacles to specialty training by limiting the capacity of staff to attend courses.

There are, however, more fundamental concerns which impact on training. One is the difference in structure and culture of the specialties of family planning and GUM. Health practitioners working in family planning and GUM come from separate disciplines and focus on different aspects of health care. Broadly speaking, family planning has traditionally been preventive in scope, largely sessional and community based, ${ }^{20}$ while GUM is predominantly more curative in approach, is seen more in terms of a career option, and is usually hospital based. ${ }^{17}$ The divide between the two specialties may be narrowing as new recruits are socialised into a more holistic ethos. Yet there is a danger that the perceived status inequalities which seem to stem from differences between the two specialties may be more resistant to change.

Some GUM practitioners question the competence of family planning staff to conduct GUM work and some family planning staff see those working in GUM as ill equipped to practise family planning. ${ }^{17}$ Concerns about diminished quality may mask more fundamental concerns about professional status, autonomy, and expertise. There are real fears of dilution of expertise among health professionals. Specialisation is seen as a key to advancement and, particularly among GUM physicians, there are anxieties that career progression might be slower in an integrated service. Enthusiasm for broadening expertise, then, is by no means universal, especially where breadth is perceived to be achieved at the cost of depth. For many GUM practitioners, their specialism is the single most attractive feature of their employment.

These more intractable aspects of the divide between family planning and GUM, as traditionally practised, clearly need to be addressed in training programmes. This problem may have been worse in the past when the gulf between the two specialties appeared wider. Change is likely to occur naturally through the upcoming stream of younger recruits. Indeed, a degree of integration may be inevitable precisely because of changes in medical education and training which are already in place.

Wider opportunities are now available to younger doctors. The introduction of the Faculty of Family Planning and Reproductive Health Care (FFPRHC) was pivotal to the major changes in the family planning specialty, particularly with respect to having a more structured career ladder. This has the potential to encourage younger doctors to see family planning in terms of a career option. Many GUM physicians have now had formal training in family planning and many are members of the Royal College of Obstetricians and Gynaecologists, ${ }^{21}$ and GUM trainees are required to obtain the DFFP. ${ }^{22}$
Significant modules in GUM are included in community gynaecology. ${ }^{22}$ Opportunities for professional development are also increasing. Family planning doctors making the transition to more holistic services are able to attend Diploma courses in GUM, nurses the English National Board course in GUM and $\mathrm{FP}^{23}$

Additionally there is now increasing cooperative training being developed around the United Kingdom with, for example, study units on sexual health being offered in some universities. There is also joint working between the FFPRHC and MSSVD such that DFFP and sexually transmitted infection foundation (STIF) courses are increasingly being run in conjunction with one another.

Despite moves in the right direction, there remains a need for shared basic level training for GUM and family planning practitioners. However facilitative the provision of integrated services, the personal perspectives of those working in each of the specialties will be important in determining their success. Training may not solve all problems associated with service integration, but it is likely to be an important determinant, not merely of the comprehensiveness of service provision, but also of the cohesiveness of the profession.

Sex Transm Infect 2003;79:354-356

\section{Authors' affiliations}

R Kane, K Wellings, Centre for Sexual and Reproductive Health Research, Department of Public Health and Policy, London School of Hygiene and Tropical Medicine, London WCIE 7HT, UK

Correspondence to: Roslyn Kane, Centre for Sexual and Reproductive Health Research, Department of Public Health and Policy, London School of Hygiene and Tropical Medicine, London WCIE 7HT, UK. Roslyn.kane@|shtm.ac.uk

\section{REFERENCES}

Fox L, Williamson NE, et al. Improving reproductive health: integrating STD and contraceptive services. J Am Med Wom Assoc 1995; 50:129-36.

2 Stedman Y, Elstein M. Rethinking sexual health clinics [editorial]. BMJ 1995;310:342-3.

3 Walsh J. Family planning provision in genitourinary medical clinics: a quiet revolution. Br J Fam Plann 1996:22:27-30.

4 Department of Health. The national strategy for sexual health and HIV. London: DoH, 2001.

5 Brook Advisory Centre. Annual Report for 1989 1990. Birmingham, 1990.

6 Singaratnum A, Asboe D, Boag F, et al. Preventing the spread of HIV infection. BMJ 1991:302:469.

7 Carlin EM, Russell J, Sibley K, et al. Evaluating a designated family planning clinic within a genitourinary clinic. Genitourin Med 1995;71:106-8.

8 Asboe D Boag F, Evans B. Women's health potential for better services. Genitourin Med 1992;68:65. 
9 Queen H, Ward HF, Smith C, et al. Women's health potential for better coordination of services. Genitourin Med 1991;67:215-19.

10 Wilkinson C, Massil H, Evans J. An interface of chlamydia testing by community family planning clinics and referral to hospital genitourinary medicine clinics. Br J Fam Plann 2000;26:206-9.

11 Eversley RB, Policar M, White V, et al. Self-reported sexually transmitted diseases among family planning clinic clients: ethnic differences in sexual risk behavior and HIV risk reduction. Ethnicity and Disease 1993:3:181-8.

12 Doherty L. New approaches to sexual health services in a rural health board area: involving service users and primary care professionals. Int J STD AIDS 2000;11:594-8.

13 Thompson C. Contraceptive needs of women attending a genitourinary medicine clinic for the first time. Sex Transm Infect 1998;74:433-4

14 Tobin JM, Bateman J, Banks B, et al. Clinical audit of the process of referral to genitourinary medicine of patients found to be chlamydia positive in a family planning service. Br J Fam Plann 1999;24:160-3.

15 Ward H, Kubba A, Bradbeer C, et al. Consensus workshop on sexually transmitted diseases and contraception: sexual health promotion and service delivery. 1995.

16 Hardee K, Yount KM. From rhetoric to reality: delivering reproductive health promises through integrated services. Family Health Int 1995:5:39.

17 Kane R, Wellings K. Integrated sexual health services: the views of medical professional. Culture Health and Sexuality 1999;1:131-45.

18 Stewart FH. Integrating essential public health services and managed care. Family planning and reproductive health as a case study. West $J$ Med 1995; 163(Suppl):75-7.

19 Greenhouse PA. Primary and secondary sexual health services need a consistent philosophy. BMJ 1995;310:1193.

20 Woolley PD. Family planning doctors should refer patients with sexually transmitted diseases to specialists. BM 1995:310:1193.

21 Nunns D, Mandall D. Most genitourinary physicians are trained in family planning. BMJ 1995;310:1194.

22 Wilkinson C, Hampton N, Bradbeer C. The integration of family planning and genito-urinary medicine (editorial). Br J Fam Plann 2000;26:187-9.

23 Jones $M$. Integrating family planning with GUM: developing a holistic sexual health clinic in Eastbourne. Br J Fam Plann 2000;26:221-4.

\section{Validity and utility of screening tests for STls}

\section{H Ward, J Weber}

\section{Implications for STI control}

T he two key parameters in defining the utility of a screening test are its sensitivity and specificity. Sensitivity is the ability of the test to correctly identify individuals with the condition; specificity is the ability to correctly identify those without. There is usually a trade off between the two. For a screening test the priority is usually to identify all those with early or asymptomatic disease at the expense of including some false positives. This way those with a negative screening test can be told with some confidence that they are not affected, and those who screen positive can be further investigated through a diagnostic test with higher sensitivity to exclude the false positives. This traditional teaching relates to programmes to detect early stages of chronic disease such as cancers and cardiovascular disease. ${ }^{1}$

In STIs, screening has an expanded role. The aim is not only to identify individuals with asymptomatic disease in order to treat and reduce sequelae, it is also to reduce transmission and contribute to STI control.

Recent developments in nucleic acid amplification techniques have revolutionised diagnostics in, for example, chlamydia screening. These have the capacity to detect tiny numbers of organisms using less invasive sampling techniques. ${ }^{2}$ This has led to an expanded gold standard compared with the earlier norm of culture, making older tests appear even less sensitive in comparison. There is pressure to base any expanded screening programme on the most sensitive tests available.

However, effectiveness of a screening programme is not based only upon the validity of individual results. It is also based on the coverage of the relevant population and on the ability to rapidly and effectively treat those who are infected in order to break the chain of transmission. One strategy for doing this is to develop tests that can be carried out rapidly with results given to the patient at the same consultation. There have been major advances in the past decade in developing such point of care (POC) tests, but almost invariably they have a lower sensitivity than the ever expanding gold standard. This means that many programme managers dismiss POC tests as inappropriate. This may be short sighted. Using tests that require laboratory support usually means that patients have to return for their results, introducing a delay of 1-2 weeks before treatment can be initiated allowing time for further transmission. In addition, some patients remain untreated as they do not return and cannot be traced. A test with an immediate result would overcome these two problems. This has been called the rapid test paradox, in which a lower rate of detection leads to more cases being treated. ${ }^{3}$ Screening for an infectious agent can thus be thought of as similar to vaccine programmes, where population immunity is a key factor in addition to individual vaccine efficacy.

In STIs screening has an expanded role...to reduce transmission and contribute to STI control

In a very useful contribution to this debate in this issue of STI, Vickerman and colleagues ( $p$ 363) report results of a modelling exercise to look at the sensitivity requirements of POC tests in relation to their potential impact on STI control. ${ }^{4}$ They use data from various populations in Africa and in the United Kingdom to inform the model, and show that a test with a relatively low sensitivity can still make a significant contribution to STI control in situations where, for example, only $80 \%$ of women return for treatment and $50 \%$ of those infected transmit to a partner during the treatment delay.

The authors are particularly interested in the implications for STI control in resource poor settings, where laboratory facilities are limited and cheap POC tests could be a useful addition to the limitations of current syndromic management approaches. But this should not be dismissed as irrelevant for wealthier countries with good laboratory facilities. In a recent study of opportunistic chlamydia screening in young people in London, with a prevalence of $10.6 \%$, only $76 \%$ of those with a positive or equivocal result returned for treatment. ${ }^{5}$ 


\section{EDITORIALS}

In mobile and hard to reach groups such as tourists, sex workers, and refugees there would be added advantages to using rapid tests. Less is known about whether a test with immediate results would be more acceptable to those who are being screened, but given the increasing popularity of instant access to information and services, it seems likely to have a widespread appeal.

Once again in the field of STI control we may be facing a conflict between the population or public health perspective, where coverage and rapid treatment is the key, and the individual or clinical perspective, where a high level of validity is paramount.

Sex Transm Infect 2003;79:356-357

\section{................}

\section{Authors' affiliations}

H Ward, Department of Epidemiology and Public Health, Imperial College London, UK J Weber, Department Genitourinary Medicine and Communicable Diseases, Imperial College London, UK

Correspondence to: Helen Ward, Department of Epidemiology and Public Health, Imperial College London, St Mary's Campus, Norfolk Place, London W2 IPG, UK;

h.ward@imperial.ac.uk

\section{REFERENCES}

1 Beaglehole R, Bonita R, Kjellstrom T. Basic epidemiology. Geneva: World Health Organisation, 1993:93-6.

2 Chernesky MA. Chlamydia trachomatis diagnostics. Sex Transm Infect 2002;87:232-4.

3 Gift TL, Pate MS, Hook EW, et al. The rapid test paradox: when fewer cases detected lead to more cases treated: a decision analysis of tests for Chlamydia trachomatis. Sex Transm Dis 1999;26:232-40.

4 Vickerman P, Watts $C$, Alary $M$, et al. Sensitivity requirements for the point of care diagnosis of Chlamydia trachomatis and Neisseria gonorrhoeae in women. Sex Transm Infect 2003;79:363-8

5 Moens V, Baruch G, Fearon P. Opportunistic screening for Chlamydia at a community based contraceptive service for young people. BMJ 2003;326: 1252-5.

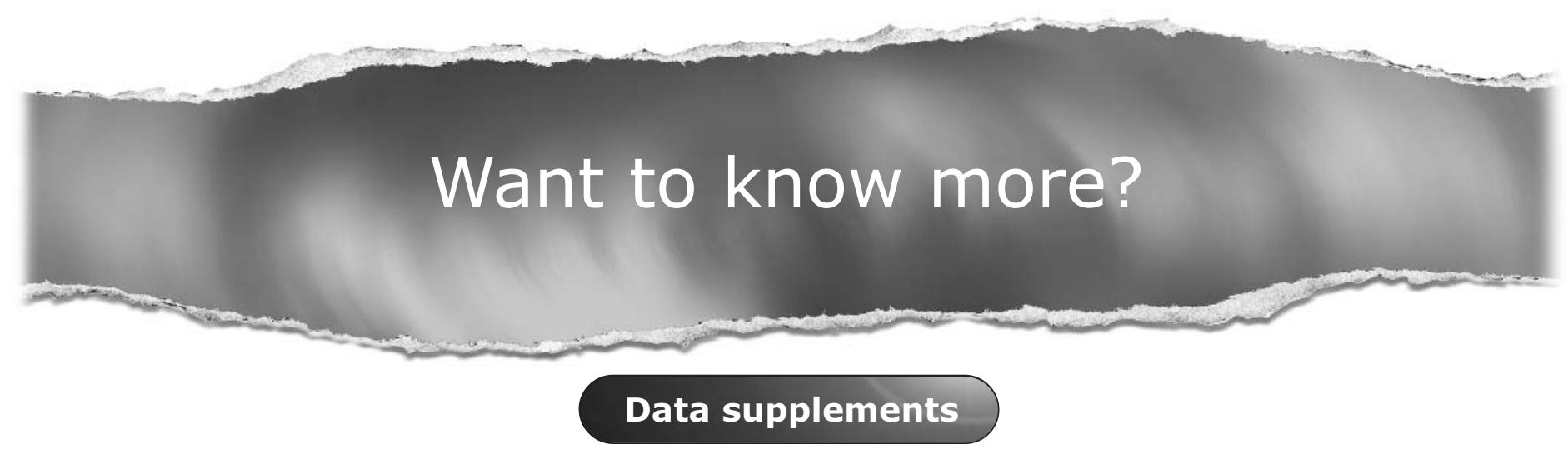

Limited space in printed journals means that interesting data and other material are often edited out of articles; however, limitless cyberspace means that we can include this information online.

Look out for additional tables, references, illustrations.

\section{www.stijournal.com}

